\title{
Tangence
}

\section{La poétique de la relation conjugale : Simone et André Schwarz-Bart dans le lit de Procuste}

\section{Catherine Wells}

Numéro 62, avril 2000

Parentèle

URI : https://id.erudit.org/iderudit/008176ar

DOI : https://doi.org/10.7202/008176ar

Aller au sommaire du numéro

Éditeur(s)

Presses de l'Université du Québec

ISSN

0226-9554 (imprimé)

1710-0305 (numérique)

Découvrir la revue

Citer cet article

Wells, C. (2000). La poétique de la relation conjugale : Simone et André

Schwarz-Bart dans le lit de Procuste. Tangence, (62), 100-122.

https://doi.org/10.7202/008176ar d'utilisation que vous pouvez consulter en ligne.

https://apropos.erudit.org/fr/usagers/politique-dutilisation/ 


\section{La poétique de la relation conjugale : Simone et André Schwarz-Bart dans le lit de Procuste Catherine Wells, Université Queen's}

En 1967, Simone et André Schwarz-Bart ont publié ensemble un roman intitulé Un plat de porc aux bananes vertes ${ }^{1}$ qui relate la souffrance d'une vieille Martiniquaise abandonnée dans un asile parisien aux allures de camp de concentration. Le sous-titre du livre, La mulâtresse Solitude, revêt une triple signification: c'est le nom d'un personnage historique à l'origine d'une révolte qui a ensanglanté la Guadeloupe au xvIII ${ }^{\mathrm{e}}$ siècle, c'est l'ä̈eule fictive de la protagoniste de ce roman à quatre mains, et c'est le titre générique d'un cycle de sept romans antillais dont Un plat de porc représente le premier tome. Ce projet collectif n'est pas encore achevé. Après la collaboration, André Schwarz-Bart produit seul un roman dont le titre reprend celui du cycle avorté : La mulâtresse solitude (1972). Simone Schwarz-Bart écrit deux romans, Pluie et vent sur Télumée Miracle (1972) et Ti-Jean L'Horizon (1979), ainsi qu'une pièce de théâtre, Ton beau capitaine (1986), avant de rédiger avec son mari un travail encyclopédique intitulé Hommage à la femme noire (1988-1989).

Dans Littérature et identité créole aux Antilles ${ }^{2}$, Mireille Rosello analyse la place ambiguë de cet auteur juif au sein de la littérature antillaise (où il fait figure d'intrus/inclus) ainsi que dans sa relation littéraire avec sa femme guadeloupéenne (où il fait figure de profiteur/collaborateur). À la différence de Dominique Malu-Meert ${ }^{3}$, qui soutient que la co-écriture romanesque représente la cristallisation littéraire d'une "conjonction, culturellement séduisante pour la mauvaise conscience européenne, de l'antisémitisme et du racisme nègre vaincus " ", Rosello remet en question les rapports de pouvoir qui régissent cette relation conjugale:

1 Simone et André Schwarz-Bart, Un plat de porc aux bananes vertes : la mulâtresse Solitude, Paris, Seuil, 1967.

2 Mireille Rosello, Littérature et identité créoles aux Antilles, Paris, Éd. Karthala, 1992.

3 Dominique Malu-Meert, Simone Schwazr-Bart, Bruxelles, Hatier, 1985.

4 Ibid., p. 7. 
Le fait est que le rapport entre Schwarz-Bart et les "Antilles" est flou, trouble, et difficilement analysable. Que dire d'un auteur, marié à une Guadeloupéenne, qui se propose de commencer un cycle de "romans antillais" et qui sollicite, pour ce faire, la collaboration de sa femme? Verra-t-on dans ce couple la reproduction de la "manufacture" familiale de Willy et Colette, où la femme, forcée d'écrire, enfermée dans sa chambre, sert finalement de "nègre" à son mari ${ }^{5}$ ?

Rosello réfute sa propre hypothèse, déclarant que la comparaison est "particulièrement risquée" étant donné la production individuelle de Simone, qui fait d'elle "non seulement un auteur à part entière, mais l'une des voix les plus fascinantes des deux dernières décennies ${ }^{6}$ ". Or, cette explication évite l'évaluation de la relation conjugale au moment de la collaboration. Bien qu'André ne soit pas le maître de Simone, il importe d'interroger les rapports qui régissent les modalités de leur collaboration. Quelques jours avant la parution du roman collectif, un article écrit par André Schwarz-Bart seul paraît à la une du Figaro littéraire ${ }^{7}$. Cet épitexte documentaire, écrit à la première personne, trace l'histoire génétique du roman du point de vue de l'auteur juif, spécifie la paternité masculine du projet initial qui naît en $1955^{\circ}$, explique le contexte discursif reliant le monde antillais au monde juif, et signale la nécessité de l'intervention féminine en 1965.

À l'époque où les lecteurs et les lectrices du Figaro littéraire lisent cet article, les deux époux occupent deux positions inégales. Entre la femme (guadeloupéenne, noire, créole) et l'homme (européen, blanc et juif), évidentes sont les différences culturelles, sexuelles, religieuses et raciales. De plus, contrairement à Simone

5 Mireille Rosello, op. cit., p. 150.

6 Ibid., p. 151.

7 "Voici le premier texte publié depuis son Goncourt (1959) par l'auteur du "Dernier des Justes": ANDRÉ SCHWARZ-BART s'explique sur huit ans de silence. "Pourquoi j'ai écrit LA MULÂTRESSE SOLITUDE" ", Figaro littéraire, Paris, $\mathrm{n}^{\circ}$ 1084, 26 janvier 1967.

8 Le projet littéraire est né de l'inspiration du moment suscitée par un commentaire fataliste d'une camarade martiniquaise ("Dans cent ans, dans mille ans, une négresse restera toujours une négresse"), ce qui lui rappelle sa propre façon de voir le monde pendant l'holocauste. Il décide de répondre à son amie, "de lui montrer qu'elle se trompait [...] par un livre". Si on ne peut pas douter de la bonne intention de l'écrivain juif, on peut remettre en question l'implication politique d'un acte discursif qui risque de réinscrire l'autorité de l'homme blanc visant à réfuter la sagesse populaire d'une femme noire. 
qui publie ici pour la première fois, André s'est déjà forgé une identité discursive lors de la publication en 1958 de son premier roman, Le dernier des justes, qui a obtenu le prestigieux prix Goncourt tout en suscitant un débat animé centré principalement sur la question de la représentation du peuple juif pendant l'holocauste. Bouleversé par le scandale, "le personnage Schwarz-Bart [qui] fascine et intrigue le Tout-Paris ${ }^{9}$ " se retira du monde littéraire pendant huit ans, jusqu'à la publication de cet article apologétique dans lequel, stratégiquement, il justifie sa représentation du peuple antillais et, significativement, pose la pierre angulaire de l'identité discursive de sa femme en définissant son rôle dans la genèse et l'écriture du roman. Je me propose d'analyser ici le paratexte d'André SchwarzBart à la lumière des théories de Linda Alcoff, Victor Segalen et Édouard Glissant, afin d'interroger sa double construction de l'Autre (du peuple antillais et de sa femme). Ensuite, une étude de la réception critique d'Un plat de porc révélera les conséquences politiques de cette histoire génétique sur le lectorat.

Dans "The Problem of Speaking for Others ${ }^{10}$ ", Linda Alcoff soutient que le "lieu" d'un auteur et le contexte discursif de son texte sont inextricablement liés à des structures d'oppression qui affectent la signification épistémologique, la réception critique et l'effet politique du message transmis. En se présentant comme celui qui comprend la spécificité culturelle d'un groupe marginalisé, qui maîtrise la vérité de sa situation, un auteur risque de réactiver la logique impérialiste qui glorifie l'hérö̈sme du sujet actif tout en réduisant au silence la résistance de l'objet passif:

La pratique qui consiste à parler pour les autres est souvent née d'un désir de maîtrise, un désir de se désigner comme celui qui comprend le plus correctement la vérité de la situation de l'autre ou comme celui qui peut se faire le défenseur attitré d'une cause juste et ainsi se couvrir de gloire et d'éloges. Le fait de parler pour les autres est souvent, quoique pas toujours, la mise sous rature et la réinscription de hiérarchies sexuelles, nationales et autres ${ }^{11}$.

9 Francine Kaufman, Pour relire "Le Dernier des justes". Réflexions sur la Shoah, Paris, Méridiens Klincksieck, 1980, p. 14.

10 Linda Martin Alcoff, "The Problem of Speaking for Others", dans Judith Roof et Robin Wiegman (sous la dir. de), Who Can Speak? Authority and Critical Identity, Urbana, University of Illinois Press, 1995, p. 97-119 (et particulièrement p. 104-105).

11 Ibid.., p. 115-116 (je traduis). 
Une des conséquences graves de la représentation de l'autre est l'intériorisation de cette vision exotique par l'autre. Jusqu'au milieu du $\mathrm{xx}^{\mathrm{e}}$ siècle, il existait une production écrite qui représentait les Antilles et les Antillais avec un regard folklorique, extérieur et européen. À l'époque de l'esclavagisme, les gouverneurs, les maîtres, les missionnaires et les grands voyageurs dépeignaient les esclaves à partir d'une vision coloniale créant ce que Maryse Condé appelle "une personnalité d'emprunt (ce don de raillerie constatée par les planteurs, cette éloquence attestée par le Père Labat, cette indifférence à la mort et ce courage devant elle attestés par tous) ${ }^{12}$ ". À partir de 1635, les premiers écrivains créoles assimilèrent les thèmes, les formes, l'émotivité et la langue des poètes français tout en adaptant les attitudes coloniales et dépersonnalisantes qui mystifiaient le peuple antillais représenté. Cette poésie mimétique s'est trouvée remplacée au début du $\mathrm{xx}^{\mathrm{e}}$ siècle par la littérature doudouiste $^{13}$ qui commence à se référer à des réalités créoles (géographiques, culturelles et linguistiques) tout en gardant une vision européenne des choses. Cette littérature colonisée ne se démarquait guère de la littérature colonialiste et ne soulevait que rarement les réalités et problèmes fondamentaux du peuple martiniquais.

Pourtant, l'histoire littéraire nous offre d'autres exemples de littérature exotique où l'intention auctoriale est noble et l'effet politique, efficace. Par exemple, dans les années quatre-vingt, les nationalistes tahitiens font de l'œuvre d'un auteur français, Victor Segalen, "le livre phare de [leur] recherche identitaire ${ }^{14}$ ". Dans son Discours sur l'exotisme ${ }^{15}$, Segalen signale que si la spécificité du Divers réside en son "incompréhensibilité éternelle ${ }^{16}$ ", l'impulsion pour comprendre cette altérité constitue un acte d'oppression. Pour créer ce qu'il appelle "une esthétique du Divers", il s'agit non pas d'assimiler l'altérité à un modèle universel mais plutôt de reconnaître, respecter et admirer son impénétrabilité:

12 Maryse Condé, La civilisation de Bossale. Réflexions sur la littérature orale, Paris, l'Harmattan, 1978, p. 26.

13 Parmi les doudouistes, il faut distinguer entre le poète exotique qui est complètement aliéné et mystifié et le poète régionaliste qui explore sa créolité à travers le regard de l'Autre.

14 John Scemla, "La dégradation du Divers", Europe, Paris, nº 696, avril 1987, p. 20.

15 Victor Segalen, Essai sur l'exotisme. Une esthétique du Divers (Notes), s.l., Fata Morgana, 1978, $96 \mathrm{p}$.

16 Ibid., p. 39. 
104

L'exotisme n'est donc pas une adaptation, n'est donc pas la compréhension parfaite d'un hors de soi-même qu'on étreindrait en soi, mais la perception aiguë et immédiate d'une incompréhensibilité éternelle. Partons donc de cet aveu d'impénétrabilité. Ne nous flattons pas d'assimiler les mours, les races, les nations, les autres; mais au contraire réjouissons-nous de ne le pouvoir jamais; nous réservant ainsi la perdurabilité du plaisir de sentir le Divers ${ }^{17}$.

Édouard Glissant, grand admirateur de Segalen, dénonce plus explicitement la violence colonisatrice qui sous-tend la notion de compréhension :

Dans la magnifique perspective des cultures occidentales organisées autour de la notion de transparence, c'est-à-dire de la notion de compréhension, "comprendre", je prends avec moi, je comprends un être ou une notion, ou une culture, n'y a-t-il pas cette autre notion, celle de prendre, d'accaparer? Et le génie de l'Occident a été de nous faire accepter cela, de nous faire accepter sans révision que comprendre, c'était l'opération la plus élevée qui puisse exister pour l'esprit humain. Et moi, je dis que ce génie est un génie trompeur, parce que dans comprendre il y a l'intention de prendre, de soumettre ce que l'on comprend à l'aune, à l'échelle de sa propre mesure et de sa propre transparence ${ }^{18}$.

Chez le maître à penser de l'antillanité ${ }^{19}$, la notion d'opacité remplace celle de transparence au sein d'une théorie qu'il forge à partir de l'idée ségalienne de l'esthétique du Divers. La "poétique de la relation" se caractérise par "la volonté d'accepter l'opacité de l'autre comme une donnée positive et non pas comme un obstacle 20 " et lutte contre l'impulsion eurocentrique de comprendre l'autre et de sublimer sa différence:

Le même, qui n'est pas l'uniforme ni le stérile [...] ponctue l'effort de l'esprit humain vers cette transcendance d'un humanisme universel sublimant les particuliers nationaux. Le Divers,

\section{Ibid., p. 25.}

18 Édouard Glissant, "Le chaos mode, l'oral et l'écrit", dans Ralph Ludwig (sous la dir. de), Écrire la parole de nuit. La nouvelle littérature antillaise, Paris, Gallimard, 1994, p. 126.

19 Aux Antilles, trois mouvements littéraires contestent l'extériorisation du peuple antillais: la Négritude proclame sa relation culturelle et mythique avec l'Afrique, l'Antillanité valorise son lien géopolitique avec les Amériques et la Créolité proclame sa spécificité anthropologique.

20 Édouard Glissant, op. cit., 127. 
qui n'est pas le chaotique ni le stérile, signifie l'effort de l'esprit humain vers une relation transversale, sans transcendance universalisante. Le Divers a besoin de la présence des peuples, non plus comme objet à sublimer mais comme projet à mettre en relation ${ }^{21}$.

Certains critiques, dont Kathleen Gyssels, Ronnie Scharfman, Mireille Rosello et Bella Brodzki, soutiennent que la littérature exotique d'André Schwarz-Bart traduit avec bonheur cette poétique de la relation glissantienne. En effet, son histoire génétique constitue en même temps un discours sur l'exotisme. Selon l'auteur, bien que le monde juif et le monde antillais soient culturellement distincts, ils sont historiquement liés par une même expérience de l'horreur: l'esclavage ${ }^{22}$. Cette relation allégorique de deux situations traumatiques (la traite des Noirs et l'holocauste) permet à l'auteur juif de relativiser l'absurdité du génocide juif en considérant l'univers concentrationnaire comme "une forme particulièrement resserrée, virulente, du monde "normal" 23 ". Ce lien devient fondamental non seulement dans la représentation de l'asile dans Un plat de porc mais aussi dans l'élaboration du cycle antillais qui s'inscrit à son tour dans un projet triptyque dans lequel chaque événement romanesque évoquerait l'univers concentrationnaire de plus en plus directement. Si Le dernier des justes a évité l'évocation explicite des camps, La mulâtresse Solitude constituerait "une approche oblique, craintive, du monde concentrationnaire ${ }^{24}$ " et un troisième ouvrage, En souvenir du vingtième siècle ou le récit de Moritz Lévy, "traiterait le monde concentrationnaire de face ${ }^{25}$. Ainsi le cycle antillais constitue une étape fondamentale dans l'évolution du survivant des camps dont "[les] livres parlent encore des Juifs, quand ils paraissent parler des Noirs ${ }^{26}$ ".

Or, pour dire l'indicible de l'univers concentrationnaire dans un roman "antillais" sans sublimer la spécificité culturelle du peuple représenté, il faut pouvoir invoquer l'insaisissable du monde antillais. L'auteur juif explique que son attirance esthétique vers

21 Édouard Glissant, Discours antillais, Paris, Seuil, 1981, p. 190.

22 Le Figaro littéraire, op. cit., p. 1.

23 Ibid., p. 8.

24 Ibid.

25 Ibid.

26 Louis Van Delft, "Les écrivains de l'exode: une lecture d'André SchwarzBart", dans R. G. Collins et John Wortley (sous la dir. de), The Literature of Exile, Winnipeg, University of Manitoba Press, 1975, p. 204. 
l'altérité antillaise précède l'insémination du projet littéraire. La description de l'artiste juif évoque "le plaisir de sentir le Divers" et témoigne de sa "volonté d'accepter l'opacité comme une donnée positive":

En fait, depuis longtemps déjà je connaissais et fréquentais les milieux antillais de Paris. Et ce qui me portait vers eux, c'était tout autre chose qu'une froide solidarité avec nos frères de couleur comme on dit maintenant. Non il s'est agit d'une grande et vive sympathie que j'ai ressentie, presque aussitôt, pour leurs façons d'être, pour leur gaieté, leur douceur, leur sagesse, leur art de vivre, et pour cette espèce de lyrisme verbal qui fait que dans la bouche d'un Antillais, d'un vrai Antillais, tout devient poésie [...]. Somme toute, comme il advient souvent, j'aimais et j'admirais les Antillais pour des qualités que je ne possède pas ${ }^{27}$.

Révolté par la critique de certains détracteurs qui remettent en question l'honnêteté de son entreprise, Schwarz-Bart défend sa position au nom d'une théorie de la communication qui dénonce la sublimation de l'autre tout en promulguant une poétique de la rencontre :

Il m'a toujours paru - il me paraît encore - que chaque humain peut légitimement parler de tous ses semblables. Je crois - ce qui n'est pas chose simple, sans doute - à l'unité fondamentale de l'espèce. Je crois [...] que l'essence du dialogue n'est pas dans les idées universelles communes aux interlocuteurs ni dans les idées que l'un se fait de l'autre, mais dans la rencontre même, dans l'invocation, dans le pouvoir qu'a le Moi de dire $\mathrm{Tu}^{28}$.

Jusqu'ici, tout semble correspondre aux théories de Segalen et Glissant. Or, lors de sa première tentative poétique, le récit autobiographique qu'une vieille Martiniquaise exilée raconte à sa petite-fille née en Europe, l'auteur prend conscience de son incapacité à évoquer authentiquement le discours de son personnage. Cette découverte fait naître sa volonté non pas d'assimiler l'autre, mais de s'assimiler à l'autre afin de mieux saisir son insaisissabilité :

Mais je savais qu'il manquait aux discours de la grand-mère une authenticité, une richesse de ton, une précision qui supposait

27. Le Figaro littéraire, op. cit., p. 1.

28 Ibid., p. 8. 
que je fasse le voyage des Antilles. Il manquait, disons, tout le fond culturel que je devinais chez mes amis antillais, sans pouvoir le saisir véritablement [... ${ }^{29}$.

En se proposant de "se faire autre pour pouvoir [...] écrire " son livre, Schwarz-Bart dévoile une volonté de prendre possession "[des] qualités qu'il ne possède pas ${ }^{31}$ ". Autrement dit, son projet littéraire présuppose une quête culturelle, la compréhension de l'incompréhensible ainsi qu'une mission anthropologique: la créolisation de l'imaginaire juif. Aidé financièrement par l'attribution du prix Goncourt et moralement par trois auteurs noirs (Alioune Diop du Sénégal, Jacques Rabemananjara de Madagascar, et Aimé Césaire de la Martinique) ${ }^{32}$, le grand voyageur occidental amorce son aventure afro-antillaise. Pendant quatre ans (1960-1963), il poursuit son immersion culturelle en retraçant le circuit triangulaire de la traite esclavagiste (Afrique-AntillesEurope). Vers la fin de l'année 1963, après avoir élaboré seul les données préliminaires (analogiques, culturelles, géographiques, historiques, mythiques et narratologiques), Schwarz-Bart rentre en Europe pour écrire seul son roman antillais. En lisant son texte, il se rend compte qu'il se retrouve au point de départ, c'est-à-dire devant un discours exotique qui n'évoque pas l'opacité culturelle antillaise :

Comme je l'ai indiqué plus haut, je croyais au pouvoir sans limites de la sympathie humaine, qui était ma seule arme en cette aventure; je découvris, soudain, que si mon livre respecte la lettre du monde antillais — peut-être même l'esprit — il en omet le parfum, ce bouquet dont on parle je crois à propos des crus délicats. Tout le possible avait été fait. Rien, me semblait-il

\section{Ibid.}

30 Ibid.

31 Ibid.., p. 1.

32 Dans l'introduction, l'auteur remet en question son droit de représenter les hommes noirs sans la permission explicite des représentants du monde noir. Il est encouragé par l'accueil généreux de ces trois auteurs avec lesquels il fait un pacte: avant de publier ce roman, il le soumettrait à un des trois afin qu'il juge de son authenticité. Ainsi, l'approbation qu'il reçoit d'Aimé Césaire en 1966 représente non seulement l'aboutissement du projet, mais aussi la marque sacrée de l'authenticité de sa représentation. Aujourd'hui, on pourrait remettre en question la sagesse d'un Européen qui demande aux Africains s'il a le droit de représenter les Créoles. En 1966, ce geste assimilateur correspond plus ou moins fidèlement à l'idéologie universaliste de la Négritude, mouvement contemporain qui tient pour acquis que, culturellement, l'Antillais est Africain. 
108

dans mon texte, ne représentait une trahison de mes frères antillais. Rien sauf, précisément, l'absence de cet indicible que je découvrais et qui est la fleur de toute ouvre jaillie d'un terroir, d'un sol spirituel [...]. Je découvris, dans l'effroi, que s'il y a toujours communication entre les hommes à des degrés divers, cette communication ne peut pas être entière, transparente, qu'à l'intérieur d'un même groupe. Je découvris, dans la désolation, qu'il est une seule façon de respecter entièrement l'originalité d'un peuple: c'est d'en faire partie. Et, pour parler plus plaisamment, il m'apparut alors et alors seulement, que si j'étais en mesure, depuis une dizaine d'années, de respirer le parfum des Antilles, de l'apprécier, de le décrire au besoin - il m'était impossible de devenir moi-même parfum ${ }^{33}$.

Cette prise de conscience se compose de trois éléments qu'il importe d'analyser séparément. La première découverte se révèle paradoxale. L'auteur annonce l'échec esthétique de son discours et dénonce la trahison de sa représentation inauthentique, sans remettre en cause son désir de comprendre l'incompréhensible de ses "frères antillais". Or, les images militaires (aventure, arme) et exotiques (parfum, fleur) qu'utilise l'auteur occidental pour parler de sa quête poétique risquent d'évoquer le paradigme colonisateur d'une conquête culturelle. L'évangélisation des esclaves fut une aventure catholique entreprise au nom de la fraternité humaine. La description exotique des Antilles faite par des missionnaires, des voyageurs, des écrivains mimétiques se caractérise par l'exaltation de la flore et de la faune des îles. Il est donc paradoxal pour cet auteur, qui dénonce férocement l'oppression coloniale de l'Occident et qui déplore l'aspect exotique de son propre discours, de se servir d'un tel langage qui semble trahir sa participation à ces deux actes (la colonisation intellectuelle et l'écriture exotique).

La deuxième découverte est tout aussi paradoxale. En révélant la nature essentielle de l'opacité culturelle, Schwarz-Bart trahit son souci de la transparence esthétique. Pourtant, il ne s'agit pas de la transcendance universalisante qui, selon Glissant, caractérise la littérature occidentale, mais d'une transparence particulière qui, selon Schwarz-Bart, caractériserait la littérature nationale antillaise et que seul le lecteur antillais comprendrait clairement. Il est important de souligner que la lente dégradation de la langue et de l'imaginaire créoles par la francisation entamée par la 
départementalisation risque de désarmer les stratégies de survie des Antillais. Si, à l'époque esclavagiste, l'opacité du discours oral antillais constituait des formes d'opposition (c'était une façon privilégiée de communiquer des messages de résistance à l'insu du maître et de ses acolytes), l'opacité de la littérature contemporaine résiste à l'universalité qui risque de diluer l'identité créole (c'est une façon de faire entendre (pas comprendre) sa voix nationale dans un monde européanisé/américanisé). Ainsi la présence dans le discours antillais de ce que Schwarz-Bart appelle "un noyau insondable qui n'exclut pas la communication mais qui en arrête les limites 34 " est sans doute une donnée positive au sein de la communauté antillaise. Dans ce contexte, ce n'est pas la découverte des limites de la communication qu'il faut remettre en question, mais la sensation d'effroi que ressent l'auteur occidental qui ne perce pas le code antillais. Or, il ne faut pas oublier le contexte discursif dans lequel s'inscrit le projet antillais de l'auteur juif. Pour cet homme hanté depuis vingt ans par l'holocauste, l'écriture de ce roman obliquement concentrationnaire représente la possibilité d'exorciser l'horreur d'une catastrophe vécue. Ainsi, on pourrait supposer que l'effroi qu'il ressent s'enracine dans un double échec: l'aveu de l'insaisissable antillais mettant en relief l'aveu de l'indicibilité de l'holocauste.

La troisième découverte représente l'échec du projet anthropologique dans la tentative de se faire autre. Encore une fois, sans remettre en question le but ultime du survivant juif, il faut relativiser la désolation de l'artiste occidental qui, malgré ses efforts acharnés, n'arrive pas à s'intégrer à une société coloniale qui s'assimile massivement et passivement à la société occidentale depuis la départementalisation de 1945. Face à cette tragédie culturelle où, sans le savoir, les acteurs noirs portent des masques blancs ${ }^{35}$ et se regardent avec des yeux occidentalisés, la volonté même de l'artiste blanc de renverser le schéma afin de porter un regard créolisé sur l'univers concentrationnaire réinscrit sa position (relativement) privilégiée. Son commentaire sur le respect d'Autrui ("[la] seule façon de respecter entièrement l'originalité d'un peuple [est] d'en faire partie") contredit la thèse de Glissant selon laquelle "pour [respecter une culture] il faut accepter que cette culture vous oppose quelque chose d'irréductible et que

35 Voir l'œuvre de Frantz Fanon, Peau noire masques blancs, Paris, Seuil, 1952. 
vous intégriez cet irréductible dans votre relation à cette culture $^{36}$ ". La notion d'appartenance semble nier la possibilité d'une poétique de la rencontre culturelle. Le plaisir de sentir le divers ("de respirer le parfum des Antilles, de l'apprécier, de le décrire au besoin") est ici remplacé par la désolation de ne pas pouvoir posséder ses qualités ("de devenir parfum"). Face à l'échec anthropologique et poétique, l'impénétrabilité antillaise devient "un obstacle" fondamental à la communication.

Prêt à abandonner son projet, Schwarz-Bart reçoit de sa femme un texte narratif relatant un événement auquel ils avaient assisté tous les deux. Ce court texte hybride, imprégné d'opacité antillaise évoquée par la transposition linguistique du créole et par la transformation esthétique du réel, témoigne du grand talent de Simone Schwarz-Bart, ressuscite le plaisir de sentir le divers et signale le début d'une collaboration littéraire que nous pouvons appeler la Poétique de la relation conjugale:

Je fus bouleversé. Je tenais dans mes mains la substance même que je cherchais vainement et qui m'était interdite. Tout ce que j'aimais chez les Antillais depuis dix ans, toutes les couleurs et tous les parfums se trouvaient là, sous mes yeux, dans ce texte d'une modestie d'autant plus émouvante qu'il ne prétendait à rien sauf à raviver ma mémoire ${ }^{37}$.

Avant de célébrer la fusion harmonique de deux imaginaires distincts dans l'unité fondamentale d'une ouvre collective, André Schwarz-Bart analyse la spécificité de l'opacité créole de sa femme, en soulignant ses influences (la tradition orale, le milieu rural, le petit peuple, la langue créole) et en expliquant l'effet esthétique suscité par l'incorporation poétique de cette opacité. Curieusement, cette analyse renverse le schéma parasitaire en révélant que la fonction principale de l'intervention féminine est de faire du bruit créole dans la transparence occidentale de l'écriture masculine. Par exemple, l'intégration des rythmes syncopés de la tradition orale créole sert à déséquilibrer l'ordonnance régulière du texte masculin:

Les textes de Simone Schwarz-Bart sont également marqués par les traditions du conte et du récit créoles. Déjà, à lire le volume que je présente aujourd'hui, on peut s'en rendre compte à cer-

36 Édouard Glissant, loc. cit., p. 129.

37 Le Figaro littéraire, op. cit., p. 8. 
taines pages qui sont affectées d'un piaffement plus proche des vieilles danses noires des Antilles que de la démarche mesurée de l'Occident ${ }^{38}$.

Cette injection de la musicalité créole correspond à une transfusion de la langue créole qui opacifie la clarté de l'expression masculine. L'auteur occidental valorise "la richesse intérieure" de ce travail langagier tout en soulignant le danger d'une entreprise esthétique qui "[...] [risque] de perdre certaines de ses attaches avec la langue française":

C'est pourquoi, avec raison, me semble-t-il, ma femme a choisi de se retrouver dans la langue créole qui est celle de ses personnages - au risque de perdre certaines de ses attaches avec la langue française. Son œuvre, de style parlé, y gagne sans doute en richesse intérieure ce qu'elle y perd au point de vue d'une certaine clarté occidentale- trop souvent mutilatrice, lit de Procuste du cœur, si j'ose ainsi m'exprimer ${ }^{39}$.

Paradoxalement, le désordre de l'opacité créole de Simone révèle la violence potentielle de sa mise en relation avec la clarté occidentale de son mari. Selon le mythe, Procuste fut un bourreau grec qui attachait ses victimes à un lit afin d'amputer les membres qui dépassaient les limites spatiales du meuble ou de distendre ceux qui ne les atteignaient pas. Si la clarté occidentale, (ce qui correspond au style écrit du texte d'André avant la collaboration), représente le "lit de Procuste du cour" pour l'opacité créole, (ce qui correspond au "style parlé" des textes de sa femme), comment se produit le métissage poétique de ces deux éléments antithétiques dans Un plat de porc aux bananes vertes? Les liaisons sont-elle dangereuses au sein du lit romanesque? Voici ce que dit l'auteur juif à propos des trois formes de collaboration :

1) Certaines histoires, certaines descriptions, certains personnages sont directement extraits, pour l'essentiel, de ces petits récits qu'elle écrit d'abord pour elle-même. Nous sommes convenus, Simone et moi, de ne donner aucune référence à ce sujet; afin qu'une vaine curiosité n'aille pas triturer un ouvrage dont nous estimons, pour le publier, qu'il est devenu profondément un. 
2) De même, dans d'autres cas, quand le récit demande une scène, un personnage ou un dialogue précis, ma femme se charge de les écrire librement, dans sa langue personnelle, avant que je ne les harmonise avec le ton général du travail. Là non plus, aucune indication ne peut être fournie aux esprits qui ignorent (ou refusent) qu'un et un font un [...].

3) Comment classer l'invisible? [...] Tel est le troisième point dont je désire vous entretenir — et qui, à certains égards, est associé aux deux précédents. Depuis que nous travaillons ensemble, nous avons consacré des centaines d'heures à la discussion. Il nous est devenu impossible, à tous deux, de déterminer à qui appartient telle idée, telle vue, telle conception philosophique, psychologique ou sociale de l'âme antillaise. Et dans le même temps que notre relation avec les Antilles se transformait, la substance profonde du projet initial s'en trouvait modifiée ${ }^{40}$.

L'écriture double est un processus paradoxal qui implique nécessairement la mise en relation de deux impulsions contraires: l'acte créateur et l'acte destructeur. André Schwarz-Bart parle de trois formes de collaboration. Les deux premières formes de la mise en relation soulignent que la fusion créatrice passent par deux actes "procustiens": la fragmentation du corps textuel féminin ainsi que la distension de sa langue esthétique. Si on considère que chaque "petit récit" de Simone représente une unité vivante avec un début, un milieu et une fin, l'extraction des histoires, personnages, descriptions de ce corps narratif symbolise le déchirement brutal de ces membres, même si leur incorporation dans le texte collectif représente une espèce de renaissance hybride. Or, le premier énoncé, écrit à la voix passive, évite d'identifier lequel des deux auteurs ampute et intègre les segments narratifs de Simone, et il serait risqué de présumer que c'est l'homme qui accomplit la mutilation procustienne du corps textuel féminin.

Inversement, le deuxième énoncé révèle l'inégalité des rapports régissant la fusion des bouts de textes féminins écrits spécifiquement pour le roman (et non pas tiré d'un corps textuel complet). D'une part, l'utilisation du terme "harmonisation" signifie un arrangement tonal qui respecte la différence sexuelle/culturelle. Pensons, par exemple, à la notion médiévale de l'harmonie 
céleste qui tient chaque corps céleste à sa place unique dans l'univers. Or, le créateur de l'univers narratif hybride où s'entrecroisent le masculin et le féminin est bien André Schwarz-Bart, et le ton général de son harmonie céleste n'est pas celui que Simone Schwarz-Bart aurait créé si elle avait écrit ce roman "librement, dans sa langue personnelle". Ainsi, l'harmonisation masculine de la différence féminine n'est pas la sublimation universaliste de la différence sexuelle, mais la mise en ordre du désordre créole.

Si l'auteur juif reconnaît la transformation fondamentale du livre masculin entraînée par l'incorporation parasitaire de fragments féminins, il est intéressant de souligner qu'il ne fait aucune allusion à l'amputation des éléments de son propre corps textuel. La troisième forme de collaboration dont parle l'auteur n'implique pas le déchirement de textes physiques mais plutôt la métamorphose de "la substance profonde du projet initial" (masculin) provoquée par la mise en relation intellectuelle des deux auteurs. Cette transformation culturelle qui entame la modification métaphysique est "invisible" et donc indivisible, à la différence des textes féminins soumis au lit de Procuste du cour. Ainsi, on pourrait soutenir que l'intervention intellectuelle et poétique de Simone Schwarz-Bart constitue le moment d'intégration de l'irréductible antillais dans le discours obliquement concentrationnaire de son mari. Ce qui demeure problématique, c'est l'inégalité fondamentale qui semble régir la modalité de cette intégration dans le texte collectif. Tout en faisant l'éloge de l'opacité antillaise de sa femme, tout en proclamant la contribution fondamentale des deux auteurs, tout en déclarant l'unité profonde de l'œuvre romanesque, André Schwarz-Bart se pose en architecte de l'extraction destructrice et de la fusion créatrice d'un corps hybride qui s'intitule Un plat de porc aux bananes vertes.

Pour évaluer la représentation qu'un auteur donne de l'Autre, il faut, selon Alcoff, étudier les effets probables et réels de son discours. Le premier effet qu'il importe d'analyser ici se produit au moment même de la publication de l'article. Une note écrite sous la signature d'André Schwarz-Bart indique que l'appareil titulaire provient non pas de l'auteur mais de la rédaction du Figaro littéraire. Ainsi la première interprétation critique du contenu de l'article est faite par le journal littéraire lui-même. Elle risque d'affecter la réception du lectorat, attiré d'abord par les deux manchettes révélatrices qui coiffent la publication de l'article sur deux pages différentes du journal: 
114

Voici le premier texte publié depuis son Goncourt (1959) par l'auteur du "Dernier des justes"

ANDRÉ SCHWARZ-BART

s'explique

sur huit ans de silence

"Pourquoi j'ai écrit LA MULÂTRESSE SOLITUDE"

APRÈS

LE

DERNIER ANDRE SCHWARZ-BART RACONTE L'HISTOIRE DE SON LIVRE

DES

JUSTES

Cette attribution titulaire signale quatre éléments qui pourraient affecter l'interprétation de l'article:

1) Elle confond le titre du roman à quatre mains (Un plat de porc aux bananes vertes) avec son sous-titre ( La mulâtresse Solitude), ce qui suscitera une certaine confusion lors de la publication, en 1972, du troisième roman de l'auteur juif ${ }^{41}$.

2) Elle trahit une stratégie de séduction en attirant l'attention sur l'énigmatique André Schwarz-Bart, tout en démontrant clairement l'inégalité discursive des deux écrivains.

3) Elle implique un travail d'analyse car, pour intituler un texte, il faut en connaître le contenu. En effet, $80 \%$ de l'article raconte l'histoire du livre de l'auteur juif, et 20\% explique l'intervention parasitaire de la femme, dix ans après la naissance du projet initial.

4) Elle pose un jugement critique sur l'autorité relative de chaque auteur en annonçant deux fois - dont une fois en discours direct rapporté — la paternité masculine du livre.

La mise sous rature de la voix de Simone dans ces titres annonciateurs se trouve parasitée par sa présence dans d'autres paratextes éditoriaux. Un court paragraphe d'introduction, qui commente le titre principal, réaffirme la paternité masculine du roman — il s'agit bien du "prochain livre d'André Schwarz-Bart" — tout

41 Je renvoie le lecteur à l'article de Bella Brodzki, "Nomadism and the Textualisation of Memory in André Schwarz-Bart's La Mulâtresse Solitude", Yale French Studies, $\mathrm{n}^{\circ} 83,1993$, p. 213-231. 
en faisant une courte allusion interrogative à la collaboration avec Simone Schwarz-Bart :

Le prochain livre d'André Schwarz-Bart, premier volume d'une suite romanesque, paraîtra dans quelques jours. Ce sera l'un des grands événements littéraires de l'année: depuis 1959, en effet, depuis son retentissant Prix Goncourt, André SchwarzBart n'a rien voulu publier. Il s'est même refusé à toute interview. Pourquoi cette longue retraite? À quoi travaillait-il en collaboration avec sa femme Simone? L'écrivain nous l'explique lui-même dans le texte que voici, le premier qu'on aura de lui depuis Le Dernier des Justes ${ }^{42}$.

À côté de ce paratexte, une photo représentant les deux auteurs assis devant leur manuscrit contredit l'affirmation de l'"origine" paternelle du roman, tout en suggérant la nature paternaliste de cette collaboration. L'homme blanc, agent actif, tient un stylo avec lequel il écrit, corrige, efface, manipule ou met en évidence un bout de texte. La femme noire, adjuvante passive, regarde attentivement. Le sous-titre, écrit en petits caractères, identifie les deux époux et avoue pour la première fois que "La Mulâtresse Solitude est signée de leurs deux noms, comme elle est le fruit, lentement mûri, de leur travail commun". Ce texte minuscule dérange l'interprétation masculiniste du message visuel et contredit directement la déclaration d'André Schwarz-Bart, inscrite audessus de la photo en caractères plus larges: "Pourquoi j'ai écrit la Mulâtresse Solitude" (je souligne).

Ce phénomène parasitaire se reproduit à la page huit, cette fois à l'intérieur du titre. Entre les deux parties textuelles de la manchette annonçant la continuité (temporelle et métaphorique) du projet littéraire masculin, Le Figaro littéraire imprime l'image de la couverture du livre collectif où le nom de Simone apparaît avant celui d'André. Si cet ordre nominal va à l'encontre de la logique alphabétique et privilégie la contribution féminine, la position de l'icône livresque entre les deux textes ainsi que le format typographique (les caractères de l'icône sont relativement très petits) constituent à la fois une mise entre parenthèses de l'autorité féminine ainsi que la réinscription problématique de la paternité masculine. Le caractère troublant du message titulaire marque un écart d'indétermination ${ }^{43}$ qui se trouve dédoublé dans la division

42 Le Figaro littéraire, op. cit., p. 1.

43 Ma traduction de "gaps of Indeterminacy", formule de W. Iser. 
thématique de l'essai en huit parties signalées par le sous-titre du titre principal "Pourquoi j'ai écrit la Mulâtresse Solitude" et sept intertitres: "Le monde concentrationnaire", "L'accordéon", "Qui parle?", "Qui était la femme Solitude", "Le parfum des Antilles", "Ma collaboration avec ma femme", et "Avec la bénédiction d'Aimé Césaire". Spatialement et paratextuellement, la contribution de Simone Schwarz-Bart se réduit à une des étapes dans l'histoire du livre de son mari juif.

Les lectrices et lecteurs du Figaro littéraire seront affectés par la totalité de l'article, c'est-à-dire par l'interaction entre paratexte et texte. Même si le roman n'a pas suscité l'intérêt prévu par le magazine en 1967, il a fait l'objet depuis les années quatre-vingt de plusieurs analyses dans deux domaines de recherche distincts: les études juives et les études antillaises. Cet épitexte aura l'effet de diviser la critique en trois groupes qui se caractérisent par la marginalisation de Simone, la mise à l'écart d'André, ainsi que l'assimilation des deux auteurs.

Dans son article "Exiled from the Shoah", Ronnie Scharfman, qui analyse le thème concentrationnaire dans Un plat de porc, soutient que la confusion relative à la paternité et au titre du roman suscitée par l'appareil titulaire de l'article est clarifiée par son contenu qui parle explicitement de la paternité masculine du roman. De plus, il dit avoir été témoin d'une déclaration de Simone Schwarz-Bart qui avoue la même chose en 1989. Ainsi, sans nier la contribution esthétique et linguistique de Simone, Scharfman soutient qu'André est le véritable auteur du livre:

Although the headline points to the subsequent confusion over both title and authorship of the novel, the content of the piece clarifies how and why Simone finally intervened in the project, and that the title refers to a whole planned cycle of novels which, in fact, was never completed. However, it is SchwarzBart's discussion of his choice of subjet, as well as the excruciating account of how he brought the project to fruition, that inform my reading of the novel in a crucial way. It explains why I have refered to him thus far in my essay as the author of Un Plat de Porc. I have also heard Simone state that he wrote the novel ${ }^{44}$.

44 Ronnie Scharfman, "Exiled from the Shoah: André et Simone Schwarz-Bart's Un plat de porc aux bananes vertes", Twentieth Century Literature, $\mathrm{n}^{\circ} 40$, 1994, p. 255. 
Louis Van Delft est moins catégorique. Il insiste sur le fait que la contribution de Simone, "intercesseur" entre l'auteur juif et l'Autre, "fut vitale ${ }^{45}$ ". Or, son article qui paraît dans deux journaux sous deux titres différents révélant les deux côtés de la mise en relation culturelle ${ }^{46}$ ne fait aucun effort pour expliquer la spécificité de cette intercession féminine. Au contraire, mise à part cette affirmation de l'intervention ethnologique de Simone, l'article se consacre à l'étude de la similarité exemplaire entre Le dernier des Justes, Un plat de porc et La mulâtresse Solitude:

Ainsi, tel l'architecte recourant toujours au même nombre, Schwarz-Bart construit selon une même géométrie ses romans. Cette immutabilité des données de base (thèmes, personnages, espace et temps, figures, organisation du récit) sur lesquelles, comme sur des piliers toujours les mêmes, s'édifie, l'un après l'autre, chaque roman, n'est certes pas propre à la création chez Schwarz-Bart seulement. Mais elle présente chez lui un caractère exemplaire ${ }^{47}$.

Contrairement à ces prises de position qui se basent sur les commentaires paratextuels, d'autres critiques résistent radicalement à l'interprétation concentrationnaire qui privilégie le rôle de l'auteur juif. Par exemple, dans son analyse de la construction identitaire dans l'œuvre romanesque de Simone Schwarz-Bart, Frederick I. Case met en évidence l'abjection absolue de la protagoniste antillaise dans Un plat de porc, sans faire référence à l'analogie concentrationnaire, principe moteur du projet littéraire. En plus, il renverse la tendance qui cherche à marginaliser Simone en mettant sous rature la place de l'auteur juif ${ }^{48}$.

In our study of Simone Schwarz-Bart, we do not attempt to make comparison with the work of André Schwarz-Bart [...] Indeed, we go as far as considering Un Plat de Porc as a novel by Simone Schwarz-Bart even though this novel was offered with her husband André. The readers of this study will understand

45 Louis Van Delft, loc. cit., p. 196.

46 Louis Van Delft. "André Schwarz-Bart: peintre de la négritude", dans Lydie Gore (sous la dir. de), Les littératures d'expression française: négritude africaine, négritude caraïbe, Paris, Centre d'études francophones, 1973, p. 128134; voir aussi, déjà cité, "Les écrivains de l'exode: une lecture d'André Schwarz-Bart".

47 Ibid., p. 203

48 En plus, il dénonce sa place parasitaire dans la littéraire antillaise en déclarant que La mulâtresse Solitude aurait dû être écrit par un écrivain antillais (p. 134). 
118

that it would be extremely and equally abitrary to attempt to isolate passages, images and ideas to be attributed to wife or husband. For the purposes of this study, SSB has produced three novels which constitute a substantial contribution to $\mathrm{Ca}$ ribbean literature ${ }^{49}$.

À la différence de Case, Fanta Toureh ne nie pas la place de l'auteur juif dans Un plat de porc aux bananes vertes, mais elle suggère le caractère négatif de son influence en révélant une double évolution (thématique et linguistique) dans les œuvres ultérieures des deux artistes. Selon Toureh, le roman collectif est exotique du point de vue de la proximité de l'objet littéraire antillais ("Une place singulière est assignée à la Martinique, elle est vue et jugée d'un autre lieu ${ }^{50}$ ") et du point de vue linguistique :

L'emploi du créole révèle deux tendances de la part des écrivains: d'une part, parer le texte d'un exotisme assez facile, avec ses liaisons, ses répétitions, son emphase qui imite celle des contes populaires; d'autre part, remanier la structure du français à partir du créole, cette dernière tendance étant à peine ébauchée dans le roman ${ }^{51}$.

Toureh démontre que cet exotisme est absent des romans ultérieurs de Simone, et encore plus présent que dans La mulâtresse Solitude qui "représente le point de vue le plus extérieur et le plus objectif sur les Antilles 52 " tout en restant "toujours éloigné de cette culture, surtout à cause de sa langue très classique ${ }^{53}$. Cette analyse implique que l'extériorité du roman collectif provient de la plume classique de l'auteur juif, même si l'on sait que la présence créole est d'origine féminine. On ne sait pas lequel des deux auteurs a décidé d'insérer des segments narratifs en créole dans le roman français.

D'autres critiques rendent compte de la distinction entre les œuvres ultérieures à la collaboration tout en les intégrant au thème concentrationnaire. Dans son étude comparatiste, Denise

49 Frederick Case, The Crisis of Identity. Studies in the Guadeloupean and Martiniquan Novel, Sherbrooke, Naaman, 1985, p. 133.

50 Fanta Toureh, L'imaginaire dans l'oeuvre de Simone Schwarz-Bart. Approche d'une mythologie antillaise, Paris, l'Harmattan, 1987, p. 38.

51 Ibid., p. 33.

52 Ibid., p. 21

53 Ibid., p. 47. 
Brahimi met en évidence les différences (narratologiques, linguistiques, ontologiques, poétiques, sexuelles, culturelles) entre les deux livres publiés en 1972 qui "[disent] le poids d'une histoire atrocement injuste sur la vie d'une femme qui en est victime ${ }^{54}$ ". Pourtant, le titre de son article "HOLOCAUSTES " reflète plus fidèlement l'expérience concentrationnaire qu'évoque l'auteur juif par son traitement allégorique de deux épisodes tragiques de l'histoire antillaise (la traite des Noirs et la révolte des Marrons ${ }^{55}$ ) plutôt que l'expérience créole de la romancière antillaise ${ }^{56}$ qui, selon Brahimi, "choisit de mettre l'accent sur le quotidien banal, celui qui constitue, si l'on peut dire, le tissu interstitiel entre les catastrophes ${ }^{57}$ ".

D'une manière semblable, le titre de l'œuvre récente de Kathleen Gyssels, Filles de solitude. Essai sur l'identité antillaise dans les (auto-) biographies fictives de Simone et André Schwarz-Bart, répond en écho au titre proposé en 1967 par André pour le cycle antillais collectif. Or, à la différence de Brahimi, qui met en évidence la spécificité de deux ouvres distinctes dont l'une "fait le saut dans l'altérité" et l'autre procède "par continuité et contiguïté ${ }^{58}$ ", Gyssels semble effacer cette différence en regroupant l'ensemble des romans des deux auteurs sous la rubrique d'une totalité hybride qu'elle appelle le discours métis schwarz-bartien:

Dans ma conquête du discours métis schwarz-bartien - métis parce qu'il porte l'empreinte de deux identités — le caractère double, aussi embarrassant soit-il aux yeux de plus d'un critique, m'est apparu comme la foncière originalité des auteurs. Celle-ci consiste à répondre uniformément à la question de l'altérité: en démontrant que ce que désire et ce que souffre l'autre est en fin de compte ce que je désire et souffre. D'où le

54 Denise Brahimi, Appareillages. Dix études comparatistes sur la littérature des hommes et des femmes dans le monde arabe et aux Antilles, Paris, Deuxtemps Tierce, 1991, p. 27.

55 L'analogie est rendue concrète dans l'épilogue du roman qui met en relation la révolte sanglante en Guadeloupe avec celle qui a eu lieu à Varsovie pendant la Deuxième Guerre mondiale.

56 Dans Héliane et Roger Toumson, "Sur les pas de Fanotte", Textes et Documents, $\mathrm{n}^{\circ} 2,1979$, p. 13-23, Simone explique que l'histoire de Télumée Miracle est la recréation fictive d'une vieille amie guadeloupéenne, Fanotte, et que le roman s'inspire de contes créoles.

57 Denise Brahimi, op. cit., p. 16.

58 Ibid., p. 24. 
mérite de l'œuvre schwarz-bartienne: sa vérité est plus universelle que proprement antillaise ${ }^{59}$.

Cette conquête de la différence révélatrice d'une vérité universelle rappelle l'impulsion dangereuse de la compréhension contre laquelle Alcoff, Glissant et Segalen nous mettent en garde. Ironiquement, selon Gyssels, qui est pourtant consciente de l'inachèvement du projet initial ${ }^{60}$ et qui mentionne les entretiens de Simone sans les citer ${ }^{61}$, cette totalité universalisante traduit avec félicité les théories de Segalen et de Glissant. Je reproduis ici un extrait de son analyse qui reprend la rhétorique du paratexte de 1967 ainsi qu'une note qui démontre clairement que le thème concentrationnaire s'applique à l'ensemble des œuvres masculines et féminines :

L'influence de la tradition et de la spiritualité juives se font sentir sans cesse par le motif concentrationnaire. Il s'y lie une profonde interrogation sur le sens de la souffrance, un réquisitoire contre toute forme d'esclavage et un virulent plaidoyer pour la dignité de l'homme, quelle que soit sa culture, sa religion ou sa race. L'heureuse alliance entre négritude et judéité traduit bien la multi-relation prisée par Glissant. Celle-ci requiert une esthétique spécifique, longuement décrite et définie par le forgeron de l'Esthétique du Divers. (p. 22)

Le premier roman renvoie le plus au monde juif : micro-univers concentrationnaire, l'inhumain pourrissoir enferme entre autres une Juive, souffrant de crises suicidaires suscitées par des souvenirs insurmontables. J'aurai l'occasion de revenir sur les parallélismes (esclavage décrit [dans Ti-Jean], vie prisonnière dans les mornes isolés [dans Télumée Miracle] et enfin, le massacre à Matouba [dans La mulâtresse Solitude]) ${ }^{62}$.

Ma lecture de la représentation de Simone Schwarz-Bart dans l'histoire génétique de l'œuvre de son mari semble indiquer l'inégalité fondamentale qui régit les rapports dans la poétique de la relation conjugale. L'étude de l'appareil titulaire de l'article et l'analyse de la réception critique appuient cette interprétation en mettant en évidence les conséquences néfastes de cette construction discursive. Il est donc très curieux de constater que, dans ses rares entrevues, Simone Schwarz-Bart n'accuse jamais son mari d'avoir mutilé son corps textuel ni d'avoir profité insidieusement

59 Kathleen Gyssels, Paris, l'Harmattan, 1996, p. 10.

60 Ibid., p. 27.

61 Ibid., p. 19.

62 Ibid.., p. 22 (en note). 
de ses talents linguistiques/esthétiques afin d'enrichir son propre projet littéraire. Au contraire, elle corrobore cette version masculine: "Je ne pensais pas du tout qu'un jour j'aurais écrit. J'ai épousé un écrivain qui avait commencé un cycle antillais indépendamment de moi. J'insiste là-dessus. Je n'ai absolument pas amené mon mari à la littérature antillaise. Après Le dernier des justes il s'est heurté à des problèmes ${ }^{63}$." Or, si elle avoue sa dette envers son mari qui fait naître sa vocation littéraire au moment de la lecture de sa petite anecdote, elle souligne en même temps son insatisfaction face aux limites imposées par l'écriture collective: "Chacun de nous [...] n'est pas allé jusqu'au bout de ce qu'il avait à dire ${ }^{64}$." Pourtant, elle signale également que la rupture ne fut pas le résultat de sa contestation féminine contre la domination masculine, mais la décision mutuelle des deux auteurs ${ }^{65}$.

Sans dénoncer directement les modalités de l'interaction poétique, les commentaires de Simone Schwarz-Bart aident à comprendre l'avortement du cycle concentrationnaire en indiquant deux sources (poétique et culturelle) de diffraction: "Un livre doit être une unité et nous avions deux façons de nous exprimer et nous représentions deux mondes. Nous étions conscients de cela et nous avons mené cette expérience jusqu'au bout ${ }^{66}$." Le projet d'André Schwarz-Bart, tel qu'il est élaboré dans le Figaro littéraire, est de relater, relier, et relayer ces deux mondes (juif et antillais) et d'harmoniser ces deux expressions (transparente et opaque) afin d'exorciser le fantasme concentrationnaire.

Au contraire, Simone, qui reconnaît le lien historique entre ces deux mondes culturellement distincts, déclare que son but est de représenter son propre peuple et de lutter poétiquement contre l'oppression de ce peuple:

Il y a une histoire de la souffrance qui est commune. Ce sont des mondes qui sont différents. Certes, le stetl, le ghetto ont disparu maintenant. Chez nous aussi ça peut disparaître, d'un moment à l'autre, et c'est dans ce but que je lutte et que j'écris. Ces petites choses qui sont tellement importantes, c'est l'âme d'un peuple ${ }^{67}$.

63 Ibid., p. 14.

64 Mary Jean Green, "Simone Schwarz-Bart et la tradition féminine aux Antilles ", Présence francophone, Sherbrooke, n 36, 1990, p. 130.

65 Ibid.

66 Citée dans Toureh, op. cit., p. 50.

67 Citée dans Green, op. cit., p. 131. 
En fait, Simone, qui dit s'inspirer plus de l'écriture de Tchékov que de celle de son mari, semble se reconnaître plus d'affinités avec le peuple russe qu'avec le peuple juif :

Le peuple russe, c'est un peuple esclave aussi, comme nous. Les grands hommes et grandes femmes de chez nous, ce sont les gens du peuple aux prises avec les mêmes problèmes dont parle Tchékov: avilissement, sentiment de déchéance contre lequel tout homme lutte sans même savoir contre quoi il lutte. Je dois à Tchékov de m'avoir fait découvrir de grands hommes dans les petites gens déchues ${ }^{68}$.

À la différence de l'expression classique de l'écriture de son mari, Simone Schwarz-Bart explique que son style parlé est la traduction linguistique de sa volonté "[...] de mettre, dans cette espèce de langue française que j'écris à ma manière, l'esprit de notre langue $^{69}$ \%. Elle dénonce la pratique consistant à incorporer des segments créoles dans le texte français comme étant un échec sur le plan de la communication ${ }^{70}$. Malheureusement, elle ne se prononce pas sur la présence "exotique" d'expressions créoles dans Un plat de porc aux bananes vertes.

Ainsi, les commentaires de Simone Schwarz-Bart n'appuient pas mon interprétation de l'inégalité des rapports régissant les modalités de la poétique de la relation conjugale mais indiquent plutôt clairement les raisons du divorce romanesque. De plus, sa récente collaboration avec son mari témoigne du rapport harmonieux entre les deux écrivains, tout en renversant la hiérarchie sexuelle. Simone Schwarz-Bart est la rédactrice principale de Hommage à la femme noire, mais elle travaille "avec la collaboration d'André Schwarz-Bart ${ }^{71}$ ”. Il est peut-être significatif que cette anthologie composée de six volumes se donne comme but de lutter contre "l'invisibilité historique de la femme ${ }^{72}$ " et de rappeler au monde patriarcal que "s'il y a eu des héros, c'est grâce aux femmes qui les ont soutenu moralement ${ }^{73}$ ".

68 Ibid.

69 Citée dans Toumson, op. cit., p. 19.

70 Ibid.

71 Christiane P. Makward et Madeleine Cottenet-Hage, Dictionnaire littéraire des femmes de langue française, Paris, Éd. Karthala, 1995.

72 Gyssel, op. cit., p. 38.

73 Ibid. 